Article publié dans :

Tcherkézoff, Serge. 2019. «Postface : Le chef océanien et la terre ». Anthropologica 61(2) : 251-260. https://doi.org/10.3138/anth.2019-0029

Postface

\title{
Le chef océanien et la terre
}

Serge Tcherkézoff, EHESS, directeur de recherches (émérite), membre du CREDO (AMUCNRS-EHESS) et ANU (Prof, hon.)

stcherk@pacific-credo.fr

st.pacific.dialogues@gmail.com

L'ensemble thématique de ce volume paraît à point nommé. Il souligne à la fois la densité des transformations en cours, qui affecte la position de «chef » dans cette région qu'on appelle Océanie ou Pacifique, et la nécessité de revoir la manière dont l'anthropologie a traité et traite encore cette position sociale. Dans ce qui suit, je m'appuie à la fois sur les articles de ce volume, sur mes propres données concernant un autre archipel voisin, Samoa, et quelques autres publications, récentes ou sous presse.

\section{L’Océanie « lointaine »}

Un mot d'abord pour définir la région dont je parlerai ici. Parler de l'Océanie tout court, ou même du Pacifique, peut induire en erreur. Ce numéro, et les comparaisons que je ferai, relèvent seulement de l'Océanie dite « lointaine » par les archéologues (le peuplement le plus récent venant de l'ouest et qui atteignit les îles qui étaient « lointaines » par rapport à l'Asie du sud-est) et dite de langues «austronésiennes » par les linguistes. On sait à quel point l'«Océanie », ainsi que les noms attribués à ses subdivisions régionales, sont une invention de géographes européens, ne reposant ni sur des regroupements linguistiques ni sur des hypothèses sérieuses de premier peuplement, mais d'abord sur une nomenclature qu'on peut qualifier de continentale par défaut, puis sur des considérations raciales.

On regroupa, à partir du $\mathrm{XVI}^{\mathrm{e}}$ siècle, dans une « cinquième partie du monde », tout ce qui restait au-delà des quatre continents déjà répertoriés ; puis, au début du XIX ${ }^{\mathrm{e}}$, on décida de 
l'appeler la partie « océanique » du monde. Dans les mêmes années, on subdivisa en distinguant des « races » suivant les « couleurs de peau », d'où l'invention de la « Mélanésie », etc. Bien plus tard, archéologues et linguistes reconnurent qu'il fallait distinguer autrement. Une partie occidentale ou « proche » (l'ouest de la Mélanésie) et une partie dite alors « lointaine » (partie orientale de la Mélanésie, Micronésie, Polynésie: famille de langues apparentées « autronésiennes ») (voir Cagnasso 2019, Douglas 2011, Tcherkézoff 2008a). Mon propos ici sera limité à cette deuxième partie.

\section{L'erreur sur la propriété de la terre et les malentendus historiques}

Dans cette Océanie-là, il est certain que, hier comme aujourd'hui, il n'est pas d'organisation sociale qui ne fasse pas une place centrale à un statut particulier, que les premiers visiteurs européens nommèrent, selon les cas, «chef» ou «roi ». Cette traduction a immédiatement orienté, jusqu'à nos jours, une interprétation massivement ethno-centrique. En effet, dans la vision occidentale, un «chef » est un homme de pouvoir : une autorité dans le domaine politique, qui certes pouvait avoir une composante « sacrée », « rituelle », etc., mais qui était la source d'une domination sur un territoire et sur la population qui s'y trouvait, la domination sur le territoire étant elle-même considérée comme un droit de propriété.

Ce fut une erreur fondamentale. Ce numéro relève ce point, mais je prendrai l'occasion de cette postface pour développer. Dans l'Océanie dont nous parlons, la terre n'est pas et n'a jamais été une propriété au sens occidental. Mais elle l'est devenue, ici ou là, à la suite de la spoliation coloniale. La terre (vanua, fanua, fenua, whenua, etc.) n'était pas une chose que l'homme peut acquérir, elle était et est encore en bonne partie une matrice qui est la source de vie et des relations sociales. Dans les langues austronésiennes, la marque grammaticale de possession diffère selon que ego est considéré à l'origine du rapport de possession ou au contraire qu'il ne l'est pas. En Samoan, « ma » récolte se dit d'une manière, de même que « mes enfants », ou tout objet acquis, ou encore « ma terre » s'il s'agit d'une terre dite de droit privé (freehold land) : ego est à l'origine de la possession. Mais « ma » terre coutumière, ou «mes parents », ou « mon titre de chef » se dit d'une autre manière : ego n'est pas à l'origine du rapport de possession.

On connaît les conséquences de ce malentendu, qui fut parfois intentionnel de la part des premiers colons et militaires occidentaux, à Tahiti (Baré 1985), à Hawaii (Linnekin 1985), 
en Nouvelle-Zélande (Orange 1987 ; Ward 1991), etc. Les insulaires pensaient accorder, parfois contre compensation, parfois de façon forcée, un droit d'usage, un droit de planter et faire pousser, un droit d'habiter mais à la manière dont fut de tous temps l'habitat océanien, c'est-àdire une structure temporaire - alors que la permanence était avec la terre ou les pierres supportant la structure. Mais les nouveaux-venus voulurent se persuader, ou en tous cas prétendre, qu'ils faisaient signer des actes de «propriété ». Le fameux traité de Waitangi en pays maori est hélas l'exemple le plus parlant et mérite qu'on s'y arrête.

Le «Résident Anglais » nommé dès 1831 persuada quelques dizaines de chefs qu'il fallait signer une déclaration d' « indépendance » pour prévenir toute spoliation (en particulier par la France). On imagine aisément que la notion n'avait guère de sens pour ces chefs. En fait, les Anglais utilisèrent simplement le mot rangatira-tanga, formé de "chef » rangatira suivi d'un suffixe de nominalisation tanga : «ce qui définit» les chefs, en quelque sorte. Bref, on disait aux chefs de dire « qu'ils étaient des chefs », ce qui ne posa aucune difficulté. Mais ceci permit au Résident de présenter ensuite une requête au roi d'Angleterre, en demandant à ce dernier d'assurer la « protection » de cette nouvelle « confédération ».

Cette manière inimitable de la colonisation anglaise - le juridisme à l'occidentale et la mise en coupe réglée, mais qui prétendent passer par l'autorité locale traditionnelle («Indirect Rule ») - allait se prolonger. Lorsque la colonisation fut ouvertement décidée par Londres en 1837, un représentant fut envoyé. Nommé «Lieutenant-Gouverneur» auprès du « Gouverneur » de l'Australie, il lui fallait s'assurer de la « souveraineté ». Comment traduire cette notion pour faire comprendre aux chefs maoris qu'ils devaient la «donner » au chef des Anglais? On se garda bien d'utiliser les termes locaux de mana, le pouvoir sacré du chef, en usage alors dans toute la Polynésie, qui signale que l'individu a en lui une parcelle de l'origine divine du monde laquelle fut elle-même à l'origine du premier ancêtre dont le nom est perpétué dans le « chef » en question et dans la terre. On se garda d'utiliser le terme de rangatira-tanga. On utilisa un néologisme, le mot kawana-tanga, de kawana qui était la translittération de «Governor ». Dans une autre phrase, le même texte assurait les chefs et leurs familles de la « possession entière et inchangée » de leurs terres ("Full exclusive and undisturbed possession of their Lands and Estates »). Les termes maoris proposés furent tino rangatira-tanga, « le corps de ce qui fait qu'un chef est un chef» et taonga. Le mot tino « corps » a un rendement métaphorique important dans toutes les langues polynésiennes ; il est pratiquement synonyme de « ensemble », « totalité », ou bien « partie locale visible de la totalité transcendante ». Le 
second mot, taonga, est ce fameux terme qui fut au centre de l'Essai sur le don de Marcel Mauss (1923-1924), désignant une catégorie de biens précieux qui alimente les échanges cérémoniels, équivalent maori des nattes fines samoanes toga (Tcherkézoff 2016).

Le point de vue des Anglais était cohérent - pour eux. La notion de "Governor » renvoyant à «government» implique que les affaires locales sont dirigées par les autorités locales, mais que la puissance étrangère a le dernier mot si elle décide d'intervenir. Mais pour les chefs maoris, à qui le traité laissait toutes les réalités qui font qu'un chef est un chef, tout son tino et toutes les valeurs sacrées attachées à son titre, tous les taonga, la notion de kawanatanga devenait une sorte de protection transcendante équivalente à celle dispensée par le créateur du monde, Tane. Tout était donc pour le mieux.

Ce que les Anglais ne savaient pas était que le premier tino d'un chef est la terre, et que la catégorie des taonga qui, aux yeux des Anglais, se limitait à quelques objets sculptés en jade et à quelques belles nattes, comprend elle aussi la terre ancestrale, comme un article récent signé d'un Maori le rappelle avec force (Tapsell 1997). Les Anglais croyaient dire aux Maoris : « vous conservez vos titres de propriété (possession), mais nous avons un droit supérieur dans l'usage des terres s'il en va de l'intérêt de la Couronne (sovereignty) ». Mais les Maoris croyaient avoir entendu un tout autre message. Ils conservaient la totalité de ce qui définit une famille et son ou ses titres de chefs. C'est eux qui restaient donc les maîtres dans les décisions sur les droits d'usage des terres.

Que reconnaissaient-ils alors derrière le kawana-tanga des nouveaux venus? Pour répondre à cette question, il nous semble qu'un autre contexte doit être considéré. Il faut remonter un demi-siècle en arrière, pour savoir comment, à la fin du XVIII ${ }^{\mathrm{e}}$, les Maoris ont interprété la nature même des Européens, quand ils ont vu ceux-ci pour la première fois. Les Européens furent considérés comme des représentants des dieux qui ont créé le monde, envoyés par eux, et/ou comme une forme spirituelle étrange mais en rapport aux ancêtres, un double déformé (Tcherkézoff 2008b). Il n’y avait donc pas de contradictions.

En effet, hier comme aujourd'hui encore là où subsiste la tenure foncière polynésienne coutumière, un chef a une autorité sur la terre en vertu des ancêtres qu'il représente, lesquels sont eux-mêmes un produit des dieux primordiaux. Si les Européens, même mortels, étaient donc des créatures venant du monde de ces dieux primordiaux, la garantie de "protection » 
offerte par le «chef» de ces créatures ne pouvait qu'être bonne à prendre et était de même nature que ce qu'on connaissait déjà.

Lancée sur ce malentendu, l'histoire qui suivit révéla une fois encore ce que « souveraineté » veut dire dans le langage colonial occidental. Quand des Maoris attaquèrent des comptoirs de compagnies qui, prétendaient-ils, ne respectaient pas le traité, le Gouverneur fit intervenir la force. On connaît la suite.

Faisons une brève escale ailleurs, aux Salomon. Un article célèbre de Daniel de Coppet raconte comment, en pays 'Are'are, certains gardiens de la coutume, prenant conscience dans les années 1970 du malentendu introduit par les Occidentaux, expliquèrent à leurs jeunes que, s'il fallait en passer par cette notion nouvelle de propriété, alors il fallait dire, et écrire sous forme de code coutumier, que ce ne sont pas les hommes qui possèdent la terre, mais que c'est la terre qui possède les hommes. Le code inscrivit : « $§ 1$. Les 'Are'are ne possèdent pas la terre. La terre possède les 'Are'are. La Terre possède les hommes et les femmes; ils sont là pour prendre soin de la terre » (de Coppet $1985: 81)$.

\section{Gardien non propriétaire : enfant-de-la-terre}

En conclusion : si la terre n'est pas propriété, le rapport du chef à la terre n'est pas non plus une propriété. Le chef est un gardien. L'existence du chef représente la pérennité de la terre clanique qui, en tant que racine et source de vie, doit continuer à être perçue comme une permanence. Et encore, en disant « l'existence du chef », il faut préciser : il s'agit de l'existence et de la permanence du titre que le corps de ce chef rend visible. Mais si, pendant un temps court ou long, le corps du chef est absent - le titre est vacant - cela ne crée pas un problème insurmontable tant que le titre est considéré par tous comme « vivant » et valide, et visible par l'existence de la terre identifiée par ce nom-titre. On en voit un exemple dans ce numéro.

Mais l'histoire coloniale fit son œuvre. Par la force au début, puis par l'acculturation à ces idées occidentales, la notion de terre-propriété est devenue pensable localement, et même une évidence. De ce fait, les discussions et même les querelles graves sont aujourd'hui fréquentes en Océanie autour du refus ou de la reconnaissance des droits de Tel ou Tel sur une terre. Il s'en suit aussi une confusion autour des droits du chef et même une ambivalence sur la nécessité même d'avoir un chef, comme on peut le voir dans les articles présentés ici. 
D'un côté, sans chef, le lien d'appartenance au groupe social perd sa consistance. On sait à quel point, et sur ce plan les choses n'ont guère changé, les insulaires du Pacifique trouvent leur identité au travers de leur appartenance à un ensemble qui est à la fois une terre-et-un-clan, que ce soit au niveau d'un clan proprement dit, d'un village, d'une région, ou même d'un pays entier. Notons en passant qu'il faut écarter cette autre notion occidentale qui voit les Océaniens, et surtout les Polynésiens, comme des gens de la mer. Grands navigateurs, grands découvreurs de nouvelles terres, certes, mais toujours à la recherche d'une terre pour s'y enraciner.

La mythologie de l'Océanie lointaine est pleine de rêves de voyages, non pas vers des mers inconnues, mais toujours vers des terres lointaines où l'on trouverait la lumière éternelle (Tcherkézoff 2003). La prise de possession d'une île consistait à défricher puis, en son centre, créer un espace consacré, que le soleil peut inonder: le malae (ou marae). Dans la Polynésie occidentale, c'était et c'est encore aujourd'hui un simple terrain dégagé, où l'on prend soin de couper à ras du sol toute l'herbe. En Polynésie orientale, c'était une imposante plate-forme de pierres. C'est là qu'on invoquait les dieux et les ancêtres et qu'on faisait les offrandes. Mais peu importe : pierre ou gazon, ce « temple » comme on a - là encore - maladroitement traduit malae / marae est le lieu racine, défriché pour que tout y soit visible, rendu existant par la lumière solaire qui se pose en cet endroit sur chacun et chaque chose.

Dès qu'on s'éloigne de ce centre que bordent les maisons cérémonielles de chaque famille, on avance vers les régions plus « obscures »; on quitte le plus domestiqué pour aller vers le plus sauvage. Vers l'intérieur des terres, ce sont les abris pour la cuisine, puis les jardins, puis les plantations au centre de l'atoll ou sur les pentes des montagnes des îles hautes, puis, dans ce dernier cas, la forêt. Vers la mer, c'est le lagon, ensuite le récif, enfin la haute mer.

Cette vision concentrique dont le centre est l'espace « lumineux » place ainsi la haute mer dans l'autre pôle, à la périphérie. Et c'est bien ainsi qu'il faut voir toutes les organisations polynésiennes, même celles des atolls et sans doute toutes les organisations de l'Océanie lointaine. Le chez-soi, le monde socialisé, est une aire de lumière établie sur une terre défrichée (qui inclut l'espace lagonaire). La mer (au large) est du côté de la « brousse », pour emprunter à l'opposition cosmologique courante en Afrique, celle qui oppose toujours le village à la brousse. 
La langue confirme largement le caractère implicite, donc général, de cette vision. En tahitien, en samoan, et ailleurs, les termes pour les passes des récifs et pour les embouchures des cours d'eau sont des termes directionnels qui s'entendent toujours pour un locuteur placé sur la terre, même quand le mot est prononcé par les rameurs d'une pirogue qui reviennent d'une partie de pêche (Tcherkézoff 1998a). Et l'on trouve partout en Polynésie l'expression cérémonielle, prononcée avec délice, d' « enfants de la terre ». Le Polynésien tient pour certain qu'il est un enfant de la terre. Mais l'expression d'«enfant de la mer» n'existe pas. En outre, on peut trouver la référence solaire, ce qui ne nous étonnera pas, puisque le monde humain commença lors de l'apparition du soleil, une fois écartés le ciel et la terre qui étaient collés. La chanson moderne la plus célèbre au Samoa occidental, qui joue pratiquement le rôle d'hymne national dans les festivals culturels, a pour refrain «nous sommes les enfants du soleil».

Pour être, il faut être enfant-de, et l'on est enfant d'une terre, laquelle n'est pas que le sol mais qui est la trace visible d'une généalogie, d'une histoire, violente ou pacifique, souvent les deux. Pour qu'une terre ne soit pas qu'un sol mais une terre-racine, il faut qu'elle soit une histoire de noms, ou l'histoire d'un nom au moins, un « titre » comme la littérature occidentale a traduit la notion, et là encore avec tous les malentendus d'une assimilation ethno-centrique, en l'occurrence une assimilation à l'ancienne féodalité occidentale.

\section{Ambivalences coloniales et post-coloniales}

D’un côté donc, sans chef, le lien d'appartenance perd sa réalité. D'un autre côté, le chef est devenu souvent un bureaucrate, un intermédiaire, auparavant avec le pouvoir colonial, aujourd'hui avec l'Etat, à la fois investi de devoir représenter la grande « tradition », la « coutume », la kastom et de devoir être le lien à la bureaucratie étatique (voir une étude en profondeur de cette bureaucratisation dans le cas ni-Vanuatu : Tabani 2019). Jouant sur l'ambivalence de la terre, racine mais parfois propriété, le chef peut être un accapareur, et le don cérémoniel qui lui était fait traditionnellement devient alors perçu comme une charge qu'on voudrait bien parfois éviter. D'où des cas, impensables naguère mais bien attestés aujourd'hui, et on en voit des exemples dans ce numéro, de communauté où le titre de chef est resté vacant à la suite de diverses difficultés à recueillir un accord sur un successeur, et où certains membres de la communauté commencent à souhaiter que la situation perdure. En effet, on apprécie de n'avoir pas à contribuer constamment à des offrandes au chef, mais on tient absolument à dire que l'on continue de chercher activement à ce que le rôle soit à nouveau rempli : non pas seulement vis-à-vis de clans voisins, mais pour soi, 
car il faut maintenir le point, même virtuel, où la communauté trouve à ancrer son existence pérenne.

J'ai la même expérience du côté de Samoa, avec un développement inattendu mais au fond prévisible, suite à la dérive du statut de la terre. Depuis que le pouvoir politique est devenu un pouvoir affairiste préoccupé de « développement» au sens occidental, disons depuis le milieu des années 1990, le gouvernement (de ce qui s'appelait alors le Samoa occidental) a tendu une oreille complaisante aux investisseurs étrangers qui demandent de pouvoir bénéficier de garanties foncières quand ils investissent pour créer une entreprise. La grande majorité de la terre étant encore aujourd'hui de statut «coutumier» ne peut être privatisée. Déjà une vente à un étranger est impossible (tant qu'il n'a pas acquis la nationalité, par mariage ou par don de la part de l'Etat). Mais, même entre Samoans, une vente n'est possible que dans la petite portion (10\%) de terres relevant du droit privé (une petite partie des droit acquis par des colons, avec les malentendus déjà évoqués, mais entérinés durant l'époque allemande).

On ne peut donc offrir à des investisseurs qu'une garantie par un bail (lease). Ce qui déjà ouvre la porte à de grands dangers, comme on le sait bien par la situation au Vanuatu où, du moins sur les côtes de l'île principale, les baux se revendent entre investisseurs, australiens et autres, créant une véritable spéculation, comme sur des actions dans une place boursière. Mais pour signer un bail il faut un signataire du côté local. On ne peut faire signer 300 membres d'un clan (et on ne peut mettre d'accord tant de personnes sur un projet particulier). De ce fait, le gouvernement samoan, faute d'avoir ouvert la porte à la privatisation des terres à l'occidentale (mais pour combien de temps encore ?), a trouvé le moyen récemment d'ouvrir un enregistrement où, pour toute terre clanique (donc de régime coutumier), le chef local peut être enregistré comme « chef gérant » ayant le droit de signer un bail.

Encore faut-il qu'il y ait un chef, c'est-à-dire que le «titre » traditionnel lié à la terre en question soit bel et bien détenu par une personne, et non laissé vacant. Dans le cas que je connais, voilà une grande famille étendue ou clan où les divers sous-clans étaient très satisfaits de la vacance établie depuis près de 20 ans, chaque sous-groupe fonctionnant de façon de plus en plus autonome, se mettant même à clôturer les parcelles autour des maisons d'habitations comme s'il s'agissait de terres privées, voilà donc ce clan soudain agité de réunions houleuses, pour décider « au plus vite » qui choisir pour être «chef», de peur que, en l'absence d'un signataire reconnu, tel ou tel chef secondaire n'aille au gouvernement de sa propre initiative, pour prétendre être le gérant de 
l'ensemble, ou même, dit-on que, le gouvernement ne s'arroge ce droit, faute de candidat local (car il s'agit de terres près de la ville et de la mer, donc à très haut rendement potentiel d'investissement). Et voilà que soudain des liens généalogiques presque oubliés sont revisités, réinvestis, que l'on va fébrilement chercher les traces écrites de tel ou tel «court case» entendu à la Cour des Terres et des Titres, le cas échéant durant l'époque allemande au début du $\mathrm{XX}^{\mathrm{e}}$ siècle. Et ceci impliquant autant sinon plus les membres de la grande famille habitant en émigration (Nouvelle-Zélande) que ceux dans les îles.

On rejoint ici un thème qui a donné lieu récemment à un colloque passionnant, dont certains papiers sont sous presse : la vigueur de la chefferie samoane (on dit là-bas : " le système matai ») dans les communautés émigrées, en Nouvelle-Zélande ou plus loin encore (voir Anae ed. sous presse). Entre autres, signalons un auteur (Fonomaaitu (Tuvalu) Fuimaono), qui, dans son article « Serving from Afar », raconte l'importance qu'il y a eu dans une famille à ce que le titre principal continue d'être porté malgré déjà deux générations parties en émigration pour travailler à l'étranger. Il raconte comme le grand père tenait à ce que son fils prenne le titre avant son départ en émigration, comment celui-ci supplia son père de pouvoir d'abord se consacrer durant quelques années à élever sa famille là-bas, tout en promettant de revenir vite pour recevoir le titre par la cérémonie adéquate, - ce qu'il fit.

Après les heures de travail à l'usine ou au bureau, et à la paroisse locale la fin de semaine après le service, on se retrouve pour ne parler que d'une seule chose : qui choisir, ou qui a été choisi pour tel ou tel titre de chef (souvent un titre peut être démultiplié et porté par plusieurs, ce sont autant de liens visibles à l'enracinement de chacun « back home», dans les îles). Rappelons aussi que pour une population samoane de quelques 250000 habitants (dans les îles et en émigration), on compte des milliers de titres de chef, suafa matai, ce qui laisse de quoi discuter. On glose à l'infini sur les qualités qu'un chef doit avoir. Ceux qui ne vivent pas proches des Samoans de Nouvelle-Zélande ont peine à imaginer à quel point la majorité du contenu des échanges électroniques échangés aujourd'hui (courriel, blogues) ne parlent que de ces questions, avec parfois des polémiques verbalement très violentes. Gros avantage de l'échange électronique : là où le ton violent, et a fortiori l'injure, faisant irruption dans une réunion formelle, peut entraîner des violences physiques graves, l'échange électronique permet la même virulence sans les conséquences immédiates.

\section{La hiérarchie n'est pas une stratification}


Autre erreur fondamentale induite par l'interprétation occidentale de la notion de chef et relevée dans ce numéro : une vision de la société stratifiée, l'élite et le peuple, une division en classes sociales, les chefs ou même les «nobles» versus les « commoners ». Un exemple parmi tant d'autres que j'ajouterai concerne la préparation de l'indépendance pour le Samoa.

Au regard du temps historique, c'est aujourd'hui anecdotique, mais il faut savoir que cette erreur a eu pour conséquence de retarder de dix années l'accession à l'indépendance du Samoa occidental. Sur le principe, il y avait un accord dès la fin de la $\mathrm{II}^{\mathrm{e}}$ Guerre : la NouvelleZélande, les conseils de chefs à Samoa qui avaient fonctionné sous le régime de la NouvelleZélande, et l'ONU. Des commissions de l'ONU vinrent donc, de 1947 à 1959, pour préparer la transition. Ces commissions étaient composées de diplomates tous acquis à l'idée occidentale de démocratie : le suffrage universel. Or voilà que les Samoans leur expliquaient, sans rien vouloir entendre d'autre, que le futur Parlement (pays divisé en une quarantaine de districts) devait être composés exclusivement de "chefs » (les matai) et que seuls les chefs pouvaient être électeurs dans chaque district. Les commissaires furent consternés de cette vision « aristocratique » (selon leur interprétation : Tcherkézoff 1998b) et tentèrent en vain, à coup de visites répétées, de faire changer d'avis leurs interlocuteurs, car, pour ces commissaires, créer un Etat libre ne reposant que sur les « nobles » était inadmissible :

Les Samoans $[\ldots]$ ont aussi une organisation politique traditionnelle. [...] diffère grandement de la démocratie représentative, $[\ldots]$ fondée sur une hiérarchie de «nobles $»^{1}$ (chefs et orateurs). Ces nobles se réunissent en conseils ou Fono [...] Chaque noble est le chef responsable d'un groupe familial [...]. (ONU $1948: 23)$

On voit bien sur quelle (sur)interprétation du système matai se fonde la réticence de la Mission. La Mission de 1947 conclut qu'il était urgent d'attendre une plus grande maturité politique avant de donner l'autonomie. La Mission qui effectua une visite en 1951 aboutit à la même conclusion pour des raisons similaires :

De plus, l'élément de démocratie qui est implicite mais en aucun cas dominant dans la structure sociale samoane doit être développé, et l'éducation politique des Samoans, particulièrement dans les zones éloignées [de la capitale], demande encore à faire de grands progrès. (ONU 1951: 6 ; notre traduction). 
La Mission de 1953 note les progrès dans le domaine économique et social mais continue de déplorer le fait que « en somme, le suffrage direct n'existe pas parmi les Samoans et la majorité des adultes, qui ne sont pas des chefs (title-holders), n'ont aucune voix au chapitre politique (no voice in government) » (ONU 1953 : 3 ; notre traduction).

C'est pourquoi, en prenant acte du «conflit fondamental» (basic conflict) entre le « concept de représentation selon la tradition samoane » et « ceux plus modernes », la Mission demande aux membres de l'assemblée constituante (qui venait de se mettre en place) de songer à des formules « suffisamment flexibles pour permettre les ajustements » que l'avenir rendra nécessaires, quand le progrès économique amènera le peuple à ne plus accepter de limiter le suffrage et la candidature à la « classe des matai » (ONU $1953: 4-5)$.

La Mission de 1956 prend acte du fait que l'assemblée constituante persiste à réclamer le système du suffrage matai. Mais - fait nouveau - elle reconnaît une certaine « démocratie » au système matai. Elle reconnaît l'attachement fort que les Samoans expriment envers le système matai mais finalement elle accepte leur affirmation disant que ce système représente une forme de «démocratie familiale ». En particulier, la Mission note que le titre de matai n'est pas héréditaire, que le porteur de titre est élu par les membres de sa aiga («famille ») et que ces derniers peuvent reprendre ce titre (ONU $1956: 6$ et 8 ).

De guerre lasse, mais ayant trouvé où tout de même affirmer un point commun avec la démocratie occidentale, l'ONU lance la phase finale. La dernière Mission (1959) met en place l'organisation du plébiscite prévu pour 1961. Le Mandat néo-zélandais du Samoa occidental devient un état indépendant en 1962, le tout premier exemple de décolonisation dans le Pacifique.

Mais cela aurait pu être acquis en 1949 si le problème de la «classe des nobles » ne s'était pas posé. Pourtant il aurait suffi d'ouvrir les yeux : un système de « noblesse » suppose que certaines familles sont « nobles » et d'autre part. Or à Samoa, il n'y avait aucun individu qui n'appartienne pas à une famille définie par un titre et donc dirigé par un chef, un «noble » -que le titre soit occupé ou temporairement laissé vacant. Bref, il n’y avait aucune famille qui ne fût pas «noble ».

Il aurait fallu, mais cela demandait davantage d'observations sur place, de voir que le lien du chef aux membres du clan est défini non par une relation de classe ou caste, mais par une relation, 
dite teu le va, «prendre soin de l'espace relationnel entre les personnes ». Sous quelle forme fautil en «prendre soin »? Là encore les mots le disent : fe-agai-ga= c'est être assis face à face, dans une relation mutuelle. Le concept, la formule, s'applique au rapport Dieu/hommes, chef $(=$ terre $=$ titre) /clan, sœur/frère. Peu de chercheurs ont vu l'importance de cette relation et surtout l'ont bien comprise, avec une grande exception: Penelope Schoeffel (1978, 1979, 1995); voir aussi Tcherkézoff (2017).

Comme il est rappelé dans ce numéro, à Fidji la relation particulière entre les clans, maîtres de la terre et « faiseurs de chefs », et le chef lui-même qui a autorité sur eux mais qui néanmoins dépend d'eux, est appelé veiqaravi, « facing each other ». Voilà encore un bel exemple de la grande unité de cette Océanie lointaine, ou du moins de cette sous-région qui inclut la Mélanésie orientale et la Polynésie occidentale, ou «Western Polynesia including Fiji ». Car à Samoa le même concept prévaut : même étymologie, même sens. C'est le mot qu'on vient de citer : feagaiga. La base aga (PPN : *haga) signifie « être face à », avec un couple linguistique préfixe-suffixe $f e \ldots i$ (+ga pour la nominalisation en samoa) qui ajoute une mutualité entre les partenaires de l'action: «se faire face » (réalise en Samoan et East Futuna) (voir Pollex : https://pollex.shh.mpg.de).

Cette « mutualité » est en fait très particulière, car elle est une hiérarchie : un terme vaut le tout de la relation, un terme englobe l'autre est l'inverse n'est jamais réalisé. A Samoa, la relation feagaiga est celle entre le chef et ses clans, mais aussi entre Dieu et les hommes, entre la sœur et le frère (et les descendants « côté-sœur » versus les descendants « côté-frère » à partir d'une génération fondatrice d'un titre de chef. La relation entre le chef et les clans de la terre est très visible à Samoa, et ce depuis les premières observations, entres les chefs appelés ali'i, disons les chefs sacrés, et les chefs-orateurs, tulāfale . Là aussi, le chef, du moins son titre, a une origine extérieure au district, alors que les titres des « orateurs » sont locaux et enracinés depuis longtemps. D'un côté, les principaux orateurs constituent un conseil dont dépend absolument le choix d'un successeur au titre de chef. D'un autre côté, leurs titres (de ces chefs orateurs) sont dits de «moindre rang » que celui du chef, et, dans le remaniement des généalogies qui survient après l'arrivée légendaire ou même mythique du chef fondateur, ces titres deviennent des rejetons collatéraux de la lignée du chef.

Mais l'histoire coloniale vint apporter ses transformations. Ce fut l'apparition récente (dernier tiers du $\mathrm{XIX}^{\mathrm{e}}$ ) d'une notion unifiée de «chef» à Samoa, et sous un nom inconnu 
ailleurs dans la région, matai. Depuis le milieu du $\mathrm{XX}^{\mathrm{e}}$, on ne parle que des chefs en tant que «matai », et Samoa devint indépendant avec un système social et électoral dit le «fa'amatai », « à la manière des chefs » (si l'on continue cette mauvaise traduction par « chef »). Mais l'étude attentive de la littérature des voyageurs et des missionnaires, depuis la fin du XVIII ${ }^{\mathrm{e}}$ siècle jusqu'à la fin du XIX ${ }^{\mathrm{e}}$ siècle, révèle une surprise. Les « matai » sont inconnus ou en tous cas absents du devant de la scène. Tous les visiteurs, sans exception, parlent de leur interaction avec les « chefs ali' $i$ » et avec les « orateurs tuläfale » qui accompagnent les ali'i. Cependant, les dictionnaires établis par les divers ordres missionnaires, protestants anglophones et catholiques francophones, incluent le terme « matai » et le traduisent comme « head of a family » ou « chef de famille »; les alii, quant à eux, sont les «chiefs » ou « chefs ».

Les descriptions du XIX ${ }^{\mathrm{e}}$ d'évènements impliquant une communauté montrent que «l'ali’i » du lieu réunit «ses tulāfale » (orateurs) et «ses groupes familiaux (maisonnées) 'äiga » pour prendre une décision affectant l'ensemble. Parmi les ali’i, les différences de rang sont manifestes, entre les ali' $i$ qui dirigent tout un district (alliance, toujours temporaire, entre divers villages) et ceux dont l'autorité se limite à leur village ; là encore, il y a une différence entre le chef ali'i qui semble tenir le premier rang dans le village et d'autres ali' $i$. Les orateurs et leurs familles travaillent les plantations et fournissent toute la nourriture. Sur ce plan, le chef ali 'i dépend d'eux étroitement. Dans un certain nombre de cas concernant des titres d'ali' $i$ ayant une autorité sur un district ou même sur un ensemble de districts, des groupements d'orateurs forment le conseil qui décidera du choix du successeur à ce grand titre d'ali'i.

Un recensement fait en 1853 par les premiers missionnaires pour l'île de Tutuila indique les chiffres suivants : quatre chefs suprêmes ali’i (pa'ia), un par "district»; une centaine de chefs ali'i dont l'autorité s'exerce surtout au niveau du village ; 160 orateurs tuläfale ; tout cela pour une population de 1800 personnes $^{2}$.

Durant toute cette période, la notion de matai ne joue visiblement pas de rôle dans l'interaction entre les communautés samoanes et les Européens. Il faut penser, d'après les dictionnaires, que le terme s'appliquait simplement aux chefs de maisonnée ; et certains de ces chefs de maisonnées pouvaient être apparemment des « orateurs » du grand chef ali 'i local. Ou bien le terme s'appliquait déjà à tous les chefs de famille, depuis les petits orateurs jusqu'aux plus grands ali ' $i$, mais avec le simple sens d'un responsable familial qui n'était pas défini par 
un titre ancré dans une terre, et le personnage n'était significatif qu'à ce niveau interne et local de la famille.

Aucune indication, si ténue soit-elle, ne permet de penser que la situation était alors ce qu'elle est aujourd'hui, où la notion de matai est la catégorie politique prépondérante, occupant tout le champ de la «chefferie » au plan national. Ce champ est subdivisé aujourd'hui entre "les matais dont le titre est de type ali' $i$ » et « les matais dont le titre est de type tuläfale », comme le disent les Samoans dans le discours quotidien et dans les textes officiels. Cette situation contemporaine est présentée de la même manière par toutes les sources de la période qui, en partant des années contemporaines, remontent jusque vers 1925.

Nous sommes donc conduits nécessairement à l'hypothèse historique suivante. Entre le dernier tiers du XIX $\mathrm{X}^{\mathrm{e}}$ siècle et le premier quart du $\mathrm{XX}^{\mathrm{e}}$ siècle, une classification ali' $i>$ tulāfale $>$ (matai) a pris une forme pratiquement inverse :

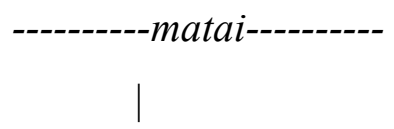

(de type) ali' $i$ - (de type) tulāfale

Le fait que deux catégories nettement hiérarchisées entre elles (ali’i et tulāfale) se soient retrouvées comme deux sous-types d'une seule catégorie (les matais) indique que ce qui faisait la valeur de l'écart hiérarchique entre ali'i et tulāfale a perdu sa raison d'être.

Tout ceci correspond à ce qu'on sait par ailleurs sur la perte de sacralité subie par les grands chefs samoans ali'i au fur et à mesure de l'entreprise missionnaire. En fait, comme dans toute situation de contact, les projections idéologiques occidentales et le dynamisme du système samoan ont interagi. A l'époque des premiers contacts et des premiers progrès de l'entreprise missionnaire, on rencontrait à Samoa des individus, hommes ou - parfois - femmes, désignés comme ali'i pa'ia et investis d'une autorité sur de vastes territoires, même si celle-ci était toujours provisoire. Ils étaient imbus d'une sacralité évidente, car leur autorité révélait qu'ils étaient dépositaires d'un grand « pouvoir ancestral ou divin » mana. Ce qu'ils avaient touché ne pouvait être touché ensuite par un homme ordinaire ; en leur présence, on se dévêtait de tout habit recouvrant le petit pagne, on se prosternait ; on ne pouvait croiser leur regard ; si on passait en pirogue dans le lagon faisant face à leur maison, il fallait se mettre à l'eau et pousser la pirogue en nageant, etc. Ces chefs étaient dits pa'ia « sacrés ». Les voyageurs et missionnaires 
européens arrivant aux Samoa retrouvaient là ce qu'ils avaient lu au sujet des grands chefs tahitiens ou hawaiiens dans les récits de Cook et de Vancouver. Ils ont cru, à tort, que ces grands chefs de districts, parce qu'ils étaient imbus de sacralité, étaient des monarques absolus. Ils les ont rapidement appelés « rois », déclenchant ainsi ce qui fera l'histoire samoane de la période 1860-1900, quand les divers «consuls» installés aux Samoa (issus de la communauté européenne locale d'aventuriers, de marchands et de missionnaires, représentant l'Allemagne, la Grande-Bretagne et les Etats-Unis) chercheront constamment, chacun de son côté, à faire nommer un seul « roi » à la tête de l'archipel, afin d'établir avec lui des traités commerciaux qui seraient valides pour toutes les îles.

En même temps, une certaine évolution de ce système a fait rapidement diminuer la sacralité de ces hommes. On constate aujourd'hui que la notion de «pa'ia» est employée uniquement à propos de Dieu, de la Bible et de la nation. Les dernières mentions de chefs sacrés datent de voyageurs de la fin du XIX ${ }^{\mathrm{e}}$ siècle. De même, l'idée traditionnelle de pouvoir mystique mana (parcelle du divin originel qui donne à l'action du chef ali'i l'efficacité nécessaire) est uniquement mentionnée aujourd'hui à propos de Dieu. Ainsi, la nouvelle religion s'est accaparé la sacralité, et la notion de chef s'est politisée.

Cette séparation se révèle dans l'interaction qu'on peut observer aujourd'hui à l'intérieur du système villageois de l'autorité, d'une part entre deux types de matais, les «ali' $i$ » et les «tulāfale », d'autre part entre les matais en général et les pasteurs.

Que s'est-il passé ? Les missionnaires du XIX ${ }^{\mathrm{e}}$ siècle ont soigneusement évité d'intervenir dans l'autorité politique des chefs. Au contraire, ils se sont placés sous leur protection. Ils y étaient contraints, étant arrivés en tout petit nombre (moins d'une dizaine), sans aucune force militaire européenne pour les soutenir. Les missionnaires ont même apporté aux chefs un surcroît de prestige et de pouvoir. En accueillant les missionnaires sous leur toit et sous leur autorité, les chefs s'accaparaient la référence à une autorité mystique supplémentaire démontrée par la possession d'objets de métal et d'armes à feu. Mais cette attitude a conduit d'emblée à tenir séparés le pouvoir religieux des missionnaires (puis celui des pasteurs locaux formés par les missionnaires) et le pouvoir politique des chefs alii.

Cette séparation fut d'autant plus nette que les missionnaires furent classés du côté des pouvoirs mystiques de la sœur d'un chef. En 1832, le chef principal ali’i pa 'ia Malietoa Vai'inupo qui 
reçut les missionnaires Williams et Barff et qui accepta le principe de la venue d'un ensemble de missionnaires qui s'établiraient à demeure (confirmant ce qu'il avait dit à Williams en 1830 lors du premier contact ; l'installation commença en 1836), classifia les missionnaires du côté des pouvoirs mystiques exercés par la «sœur» de tout grand chef. A cet effet, il leur donna comme titre honorifique l'expression «Comme-une-feagaiga-céleste ». Nous retrouvons le mot «feagaiga » qui désigne à la fois la relation frère-sœur et la sœur elle-même (mais il ne peut servir à désigner seulement le frère), quand il y a une formalisation cérémonielle entre le nom-titre fondateur de chef et un nom-titre porté par la sœur ou la fille du fondateur et qui en vint à désigner aussi la relation entre le missionnaire et la communauté (avec ses chefs), et tout simplement entre Dieu et les hommes.

La règle était alors que les descendants appartenant à ce " côté-sœur » ne réclament jamais le droit de porter le titre de chef. Mais ils avaient une sorte de droit de veto sur le choix du successeur issu des diverses branches du «côté-frère », un droit renforcé par divers pouvoirs de malédiction dont on les croyait capables (Schoeffel 1979). Tout cela reposait sur une croyance fondamentale. On considérait que le «côté-sœur» était relié plus directement à l'origine et pouvait communiquer avec les dieux et les ancêtres par la pratique du tapua'i. La relation feagaiga est un «covenant», comme les intellectuels Samoans le disent en anglais, mais ce « contrat» est supra-humain. Par la «nature » $(a g a)$ des relations humaines ( $v a)$, dans l'« essence » même de ces relations, le côté-frère est lié par ce « contrat » à un côté-sœur.

On ne dispose pas de commentaires de l'époque sur cette classification décidée par Malietoa Vai'inupo, mais on peut penser qu'elle répondait à une précaution. Ce chef était occupé à asseoir par la guerre son autorité sur une bonne partie des îles occidentales. Il vit tout le profit qu'il pouvait tirer de ces nouveaux pouvoirs, mais il s'arrangea pour que ces derniers ne lui fassent aucune concurrence sur le plan de son autorité de chef (pule), tout en se réservant pour lui-même, de ce fait, la « bénédiction » si l'on peut dire qui émane toujours d'un « côté-sœur » ${ }^{3}$. La précaution n'était pas inutile quand on compare avec le royaume de Tonga où les missionnaires méthodistes parvinrent à devenir, aux côtés du roi, les dirigeants du pays et les instigateurs d'une constitution nationale, en 1875, qui allait modifier profondément l'organisation sociale (Campbell 1992 ; van der Grijp 1993 ; Douaire-Marsaudon 1998).

Le mot feagaiga désignait également la relation entre un chef ali'i et ses orateurs tuläfale. Significativement, la même évolution qui réduisit la notion de sacralité $p a$ 'ia des grands ali'i 
au profit des missionnaires et de leur dieu, produisit une réduction de l'invocation de la notion de feagaiga pour le rapport ali'i/ tuläfale au profit de la relation entre un pasteur et son village (Schoeffel 1995). La séparation des pouvoirs entre le missionnaire (puis le pasteur local) et le chef est comparable sur bien des points à ce qu'était, avant le contact, la relation entre l'autorité sacrée de l'ali'i et le pouvoir profane des groupements d'orateurs tuläfale. De ce fait, avec l'établissement de la sacralité de la nouvelle religion des missionnaires (et donc avec la captation de la notion de $p a$ 'ia), la distinction entre ali'i et tuläfale a perdu une partie de son contenu. C'était une distinction entre le sacré et le politique. Le sacré ayant été capté par le nouveau Dieu et ses missionnaires, la distinction entre les deux sortes de chefs (entre les deux sortes de noms-titres) est devenue une simple inégalité, mesurable et reposant sur l'ancienneté : le chef avec une généalogie plus ancienne (les ali'i) versus le chef dont l'origine est plus récente (les tulāfale). En effet, les généalogies des titres d'orateurs tulāfale commencent en général par une création effectuée par un ali'i.

Il s'est constitué ainsi petit à petit l'idée d'une catégorie de «chefs-en-général » - les mataiunissant dans une même définition ceux qui étaient auparavant si fortement distingués : les ali 'i et les tuläfale, les chefs sacrés et les orateurs, ou selon d'autres termes dans des sociétés voisines, la chefferie et les gens de la terre. Cette nouvelle définition unitaire consistait dans l'idée du pouvoir profane détenu par tout chef de famille, tout leader, tout aîné. Apparemment, le terme «matai» qui désignait chaque aîné de maisonnée fut retenu pour désigner cette nouvelle catégorie générale. Mais il ne s'imposa vraiment que dans les dernières années du $\mathrm{XIX}^{\mathrm{e}}$ siècle.

\section{Conclusion}

Si l'on veut retracer, suivre et comprendre comparativement les transformations qui ont affecté et affectent le statut de Chef dans l'Océanie lointaine, l'outil qui utilise la distinction hiérarchie/stratification permet de mieux comprendre les transformations à l'œuvre aujourd'hui et qui prolongent celles des débuts de la colonisation. Plus la hiérarchie s'efface, plus elle apparaît limitée à une stratification, plus les conflits de pouvoir remplacent la stabilité des relations de respect. En disant stabilité, il faut préciser : il est clair que de tous temps, la force, dont la forme ultime était la guerre, prenait le devant de la scène dès que l'on sortait des relations de « respect». Mais chacun en était bien conscient et les deux mondes relationnels étaient bien cloisonnés. Aujourd'hui, la frontière est devenue brouillée, et l'on hésite à chaque 
pas : est-on dans le respect (dans la hiérarchie), où l'on ne gagne que par la parole, ou même le silence, mais un silence qu'on laisse planer comme une émanation du plus haut mana, qui en impose et fait en sorte que, à la fin de la réunion, après la communion cérémonielle par la boisson de kava, chacun se sent partie prenante de la décision obtenue, quitte à tenter prochainement d'en imposer à son tour pour modifier le cours des choses ? Ou bien est-on dans la confrontation, où la violence verbale et même physique est toute proche d'éclater mais où chacun a le sentiment que ce serait « une honte» pour tous les participants que de franchir ce fossé ?

Tant que cette barrière de la honte qui intervient s'il y a « manque de respect » subsiste, l'organisation des «chefferies » océaniennes restera différente des systèmes de pouvoir du monde occidental contemporain. De la même manière, de nombreux Samoans expliquent qu'il n'est pas besoin d'avoir des forces de police dans les villages tant que la notion de « respect» ( $f$ 'áaloalo) aux chefs (les divers chefs de clan, chaque village comptant plusieurs dizaines de clans) s'impose d'elle-même par cette barrière de la honte. Mais ils disent aussi que, en plus de la honte, il importe de maintenir la menace d'être banni de sa terre par le conseil du village, pour avoir commis à répétition des fautes graves de manquement à ce « respect». Or ceci à son tour ne tient que dans un régime de terres coutumières. Le jour où le gouvernement aura cédé aux développeurs et ouvert la porte à une privatisation de la terre, en propriété individuelle, cette menace n'aura plus lieu (seul l'Etat sera au-dessus de la notion de propriété individuelle). Nous voilà ramenés à la clé de voûte qui soutient toute la chefferie «océanienne » : la terre comme enracinement et source de vie, loin de l'idée occidentale de propriété privée.

\section{Bibliographie}

Anae, Melani, (dir.), sous presse. « Samoan Transnational Matai : Experiences of fa'amatai from diverse perspectives », Pacific Dynamics-Journal of Interdisciplinary Research, 3 (1).

Baré, Jean-François, 1985. Le malentendu pacifique. Des premières rencontres entre Polynésiens et Anglais et de ce qui s'ensuivit avec les Français jusqu'à nos jours. Paris, Hachette (Histoire des gens).

Cagnasso, Richard, 2019. L'apport des écoles italiennes dans les premières cartes de l'Océanie. Thèse de doctorat de l'EHESS (Sciences sociales Marseille, CREDO). 
Campbell, Ian C.,1992. Island Kingdom : Tonga, Ancient and Modern. Christchurch, University of Canterbury Press.

Coppet, Daniel de, 1985. « ...Land owns people. In honour of the late Aliki Nono'ohimae Eerehau ». In R. H. Barnes, D. de Coppet, R. J. Parkin (dir.), Contexts and Levels :

Anthropological essays on hierarchy, p. 78-90. Oxford, Anthropological Society of Oxford (Occasional Papers, $\mathrm{n}^{\circ}$ 4).

Douglas, Bronwen, 2011. « Geography, raciology, and the naming of Oceania », The Globe, Journal of the Australian and New Zealand Map Society, 69 : 1-28.

Douaire-Marsaudon, Françoise, 1998. Les premiers fruits. Parenté, identité sexuelle et pouvoirs en Polynésie occidentale. Paris, CNRS Editions / Editions de la Maison des sciences de l'homme.

Fonomaaitu, Tuvalu Fuimaono, sous presse. « Tautua ai taumalele! Serving from afar! ». In Melani Anae (dir.) . « Samoan Transnational Matai : Experiences of fa'amatai from diverse perspectives », Pacific Dynamics-Journal of Interdisciplinary Research, 3 (1).

Grijp, Paul van der, 1993. Islanders of the south: production, kinship and ideology in the Polynesian kingdom of Tonga. Leiden KITLV Press, Royal Institute of Anthropology and Linguistics, Verhandelingen Series $n^{\circ} 154$.

Linnekin, Jocelyn, 1985. Children of the land: exchange and status in a Hawaiian community. New Brunswick (N.J.), Rutgers University Press.

Mauss, Marcel, 1923-1924. «Essai sur le don. Forme et raison de l'échange dans les sociétés primitives », L’Année Sociologique, seconde série.

ONU, 1948, « Report to the Trusteeship Council by the United Nations Mission to Western Samoa - Rapport adressé au Conseil de Tutelle par la Mission des Nations-Unies au Samoa-Occidental ». New York : United Nations - Nations-Unies (Trusteeship Council - Conseil de Tutelle, Official Records - Procès-verbaux officiels, session 2, special supplement 1). 
, 1951, «Report on Western Samoa by the United Nations Visiting Mission to Trust Territories in the Pacific ». New York : United Nations (Trusteeeship Council, session 8, supplement 5).

_, 1953, « Report on Western Samoa by the United Nations Visiting Mission to Trust Territories in the Pacific ». New York : United Nations (Trusteeeship Council, session 12, supplement 5).

Orange, Claudia, 1987. The Treaty of Waitangi. Wellington, Allen et Unwin/Port Nicholson Press.

Schoeffel, Penelope, 1978. « Gender, status and power in Samoa », Canberra Anthropology, 1 (2) : 69-81.

_ 1979. Daughters of Sina. A study of gender, status and power in Western Samoa. Thèse de doctorat, Australian National University, Canberra.

, 1995. "The Samoan concept of feagaiga and its transformation ». In J. Huntsman (dir.), Tonga and Samoa : Images of Gender and Polity, p. 85-106. Christchurch, University of Canterbury.

Tabani, Marc, 2019. « Tannese Chiefs, State Structures and Global Connections in Vanuatu », The Contemporary Pacific, 31 (1) : 65-103.

Tapsell, Paul, 1997. «The flight of pareraututu: an investigation of taonga from a tribal perspective », Journal of the Polynesian Society, 106 (4) : 323-374.

Tcherkézoff, Serge, 1998a. «Mua / Muri : ordre, espace et temps en Polynésie. Le cas samoan comparé au tahitien et le rapport à l'Occident ». Bulletin de la Société des Etudes Océaniennes, $276: 27-51$.

1998b. « Is aristocracy good for democracy? A contemporary debate in Western Samoa ». In Jürg Wassmann (dir.), Pacific Answers to Western Hegemony : Cultural Practices of Identity Construction, p. 417-434. Oxford, Berg.

__, 2003. FaaSamoa, une identité polynésienne (économie, politique, sexualité).

L'anthropologie comme dialogue culturel. Paris, L'Harmattan. 
,2008a. Polynésie/Mélanésie : l'invention française des « races » et des régions de

l'Océanie. Papeete, Au Vent des Iles.

_ 2008b. First Contacts in Polynesia : The Samoan case (1722-1848). Western

misunderstandings about sexuality and divinity. Canberra, ANU Press.

,2016. Marcel Mauss à Samoa : le holisme sociologique et le don polynésien. Marseille, pacific-credo Publications (Aix-Marseille Université-CNRS-EHESS).

, 2017. «The Samoan Village, the Brother-Sister Relationship and the Rule of

Exogamy », Journal of Samoan Studies, 7 (2) : 6-36.

Ward, Alan, 1991. «Interpreting the Treaty of Waitangi : the Maori resurgence and race relations in New Zealand », The Contemporary Pacific, 3 (1) : 85-113.

${ }^{1}$ Les guillemets sont dans le texte original. Ce rapport est bilingue, anglais-français. Le passage correspondant de la version anglaise dit : « a hierarchy of " title-holders " (chiefs and orators) ». La traduction française officielle de l'époque nous montre bien quelle était l'interprétation de la notion de «title-holder » par la Mission onusienne : « hiérarchie de nobles... l'autorité de la noblesse ».

${ }^{2}$ Le recensement est présenté en un tableau de deux pages, dans une lettre envoyée au quartier général de la Mission à Londres par Thomas Powell (lettre du 12 juillet 1854, LMS archives, South Seas Letters, Incoming letters, Box 25, Folder 7).

${ }^{3}$ Sur les pouvoirs de bénédiction - et de malédiction, bien évidemment - du côté-sœur, attestés au XIX ${ }^{\mathrm{e}}$ siècle, voir Schoeffel $(1978,1979,1995)$. 